\title{
论文
}

\section{基于异构数据模型的电-气耦合网络状态 感知方法研究}

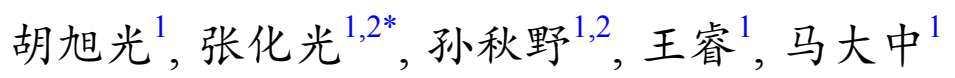

1. 东北大学信息科学与工程学院, 沈阳 110819 ;

2. 东北大学流程工业综合自动化国家重点实验室, 沈阳 110819

* E-mail: hgzhang@ieee.org

收稿日期: 2019-03-06; 接受日期: 2019-06-18; 网络版发表日期: 2019-09-17

国家重点研发计划(编号: 2018YFA0702200)、辽宁省“兴辽英才计划”项目(编号: XLYC1801005, XLYC1807009)、国家自然科学基金创新研 究群体项目(编号: 61621004)和国家自然科学基金(批准号: 61433004, 61773109, 61573094)资助

摘要 随着电力、天然气网络开放互联, 多能流耦合在实现能源科学管理与优化调度的同时, 彼此间能量转换使 得原本独立能源网络的状态感知复杂度增加. 因此, 在实现快速分析及考虑耦合网络特性的基础上, 本文提出一 种针对多维电-气耦合网络的异构数据模型状态感知方法. 首先, 针对子网络交互影响以及相应检测变量变化过 程, 构建出刻画不同子网络时间响应特性的异构数据模型. 然后将构造的模型通过归一化及函数转换得到对应的 协方差矩阵提高节点数据变化灵敏度, 并且将状态感知判断转化为随机矩阵谱差异值变化程度的分析. 在确定网 络状态改变的前提下, 根据谱分布对应的最大特征向量得到发生变化的网络节点, 从而完成整个耦合网络状态感 知过程. 最后对不同电-气耦合网络状态改变情况进行仿真, 仿真结果验证了本文所提方法的可行性与有效性.

关键词电-气耦合网络, 多时间尺度, 异构数据模型, 数据驱动, 谱分析

\section{1 引言}

随着人们对能源危机的重视及新技术、新设备的 不断发展和应用，多能耦合逐渐成为应对能源日益枯 竭现状的最优选择 ${ }^{[1 ~ 3]}$. 在此背景下, 传统电网的基本 架构也在不断改进和演变，以期在原有网络的基础上 构建一个高效能、安全可靠以及灵活多变的多能网 络，实现多能源的规模化利用，促进不同类型能源的 源、网、荷深度融合互补和紧密互动，进而提高经济 和社会效益.
近年来, 燃气轮机和电转气(power to gas, $\mathrm{P} 2 \mathrm{G}$ )的 发展进一步促进了电力网络与天然气网络的融合. 因 此, 电-气耦合网络受到了广泛关注 ${ }^{[4]}$. 与传统电力网 络相比, 电-气耦合网络存在两种不同类型能量的相互 转换、耦合和影响, 并且多维异构、区域交互以及多 时间尺度特点引发的一系列问题亟待解决 ${ }^{[57]}$. 文献 $[8,9]$ 按照不同网络的时间尺度特性, 采用奇异摄动理 论对耦合网络进行建模并且反映子系统间的相互影 响. 文献[10]研究了天然气动态模型, 并且在此基础上 提出可变压缩比的电-气耦合网络稳定控制方法. 从能

引用格式: 胡旭光, 张化光, 孙秋野, 等. 基于异构数据模型的电-气耦合网络状态感知方法研究. 中国科学: 技术科学, 2020, 50: 346-360 Hu X G, Zhang H G, Sun Q Y, et al. Research on situation awareness of electricity-gas coupling network based on a heterogeneous data model (in Chinese). Sci Sin Tech, 2020, 50: 346-360, doi: 10.1360/SST-2019-0087 
量管理角度出发, 稳态条件下的耦合网络运行也受到 了人们的关注 ${ }^{[11]}$. 文献[12]通过与电力网络进行类比 建立了天然气网络模型, 构建了电-气耦合网络稳态分 析求解方法. 文献[13]在考虑电-气耦合元件特性的基 础上, 建立了具有不同耦合设备的电-气网络稳态估计 模型. 文献[14]从能量流动的角度实现了对电-气统一 模型的建立. 与此同时, 针对综合能源系统多能流耦 合特性, 已有文献采用加权最小二乘法建立多能量测 方程和状态估计模型. 为保证天然气系统状态估计收 玫性, 文献[15]采用回路法实现稳态计算, 虽然该方法 对初值不敏感、收玫性好, 但对于含燃气驱动压缩机 的天然气网络的建模较为复杂. 相较于回路法来说, 节点法虽然能够建立形式简单的综合能源系统方程, 但是对系统初值的依赖性高. 因此文献[16]通过选取 天然气节点压力初值为管道两端压力差的 $5 \% \sim 10 \%$, 从而解决牛顿法迭代时初值选取问题. 基于遗传算法 的全局搜索能力, 文献[17]进一步研究了天然气系统 的初值问题. 在解决天然气系统初值选取基础上, 文 献[13]通过建立的电-气耦合网络稳态状态估计方法实 现了非全量测下多坏数据的辨识. 进一步地, 为了消弭 综合能源系统中的不良数据, 文献[18]通过加权最小 绝对值实现了双线性抗差状态估计, 并且通过仿真验 证了该方法具有良好性能和较高计算效率. 在上述研 究基础上, 由于不同能源管理供应区域及多能系统量 测精度差异, 稀疏通信网络下的分布式状态估计算法 仍需进一步研究以满足不同耦合能源间快速性与可靠 性要求 ${ }^{[3,6]}$. 此外, 还需要考虑天然气网络和热力网络 的动态特性, 提出多能流动态状态估计方法并且进一 步研究具有两种以上不同能源类型的综合能源系统状 态估计 ${ }^{[19]}$.

上述文献通过详细数学模型刻画了电-气耦合网 络, 但是电-气耦合网络存在的耦合元件会对系统产生 非线性、不确定性的影响, 使得耦合网络模型难以精 确量化, 同时耦合元件会对原本相互独立的不同网络 产生交互影响, 进而增加整个系统运行的危险及脆弱 性 $^{[20 \sim 23]}$. 除耦合元件以外, 由于电-气耦合网络涉及不 同类型节点、分布地域分散, 节点时刻面临着来自不 同网络及环境的影响, 会使检测数据产生不同的变化 情况. 如果状态变化未能及时发现会影响网络的正常 运行, 甚至于产生大规模断电及停气事故的严重后

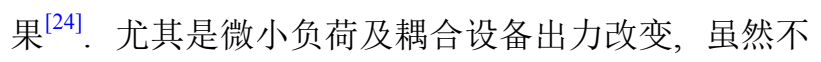

会造成网络短时间内剧烈变化及设备的可见损耗，但 是可能会加大耦合网络运行风险 ${ }^{[25 ~ 27]}$. 因此为了保证 电-气耦合网络的安全高效运行, 实现多能互补的目 标, 亟需发展面向电-气耦合网络的状态感知, 研究由 于不同物理特性网络节点改变导致的检测数据变化问 题 ${ }^{[6,19]}$.

基于上述研究情况, 本文以电-气耦合网络为研究 对象, 从检测变量角度出发, 通过研究多能流检测数据 的差异性, 实现相关信息流的集成, 完成充分反映不同 子网络检测变量异构数据模型的构建. 进而提出考虑 耦合网络特性的感知方法及相关评价指标, 从而形成 完整的状态感知分析流程, 保证不同类型节点变化检 测的准确性和有效性. 最后探讨了负荷调整以及耦合 设备出力改变对相应子网络以及与之存在耦合关系的 其他网络的影响, 为后续耦合网络的运行决策与设备 操作提供可靠数据.

\section{2 异构数据模型}

\section{1 状态感知多时间尺度划分}

不同运行特性的电力网络和天然气网络的交互使 得电-气耦合网络中存在多时间尺度特性. 根据网络传 播特性, 将相关物理变化过程及检测变量特性按照时 间尺度进行划分, 并且相对应网络时间尺度的划分结 果如图1所示.

电力网络状态变化通常由电负载及电源出力改变 引起, 而且变化及网络传播时间较快, 通常在秒级内完 成; 由于天然气网络具有大时延特性, 因此通常需要数 十秒才能完成整个网络的变化过程. 而对于耦合设备 来说, 由于其连接不同时间特性的网络, 所以变化情 况介于两个网络时间尺度之间.

在电力网络当中, 除了节点电压常被用于电力网 络运行的检测变量以外, 根据电力网络节点负荷特性 可知, 相角也可以精确地反映系统稳定性和描述电力 系统运行状态的变化, 实现电力网络稳定监测与控制. 因此相角也可以作为电力网络变化的检测变量.

\section{2 异构数据模型构建}

根据图1所示, 电-气耦合网络的多时间尺度使得 检测难以在同一时间刻度下完成, 同时两种不同能流 网络使得各系统模型不同, 特性差异大. 因此本文依 


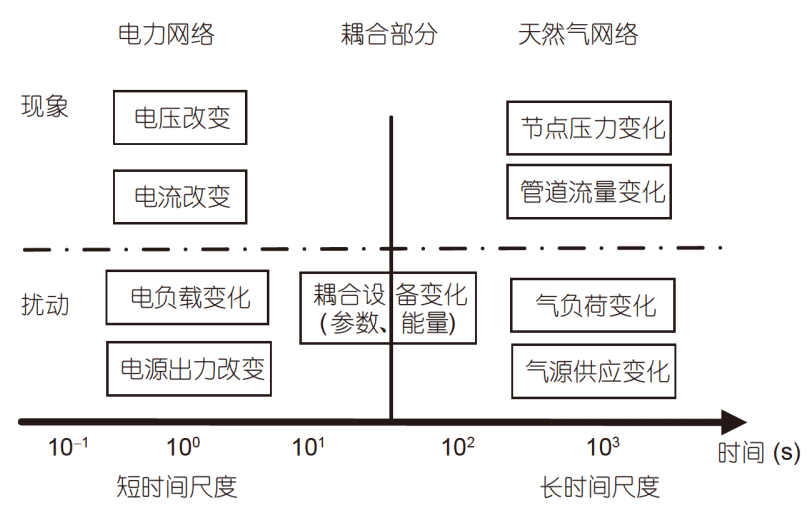

图 1 状态感知多时间尺度划分情况

Figure 1 Multi-time scale partition of situation awareness.

据耦合网络实际情况相对应地提出异构数据检测模 型. 电-气耦合网络是具有多时间尺度特性的物理系 统, 从奇异摄动理论出发, 分别用快变系统、慢变系 统两种不同模型描述不同时间尺度的电力子网络和天 然气子网络. 因此, 在不同时间尺度范围内采用时标分 解原则划分为短尺度数据模型和长尺度数据模型, 从 而有效地刻画网络变化特性 ${ }^{[8,9]}$. 采用短尺度数据模型 分析时, 认为慢变系统 (天然气子网络)检测变量是不 变的, 所以短尺度数据模型由电力网络的节点电压 $v^{x}$ $\left(x=1,2, \ldots, n_{e}\right)$ 构成; 构建长尺度数据模型时, 快变系统 (电力子网络) 的检测变量已经达到稳态, 所以长尺度 数据模型是由电力网络的节点电压 $v$ 和天然气网络的 节点压力 $p^{y}\left(y=1,2, \ldots, n_{g}\right)$ 组成.

为了便于表达及后续状态感知, 在模型构建中采 用检测变量 $s^{j}$ 表示不同时间尺度下的节点电压 $v^{x}$ 和压 力 $p^{y}$. 具体构建过程如下.

假设在采样时刻 $t$, 网络节点的检测变量 $s^{i}$ 的测量 值为 $s_{t}{ }^{i}$, 经过长度为 $T$ 的采样时间后, 检测变量 $s^{i}$ 的检 测序列为

$\mathbf{s}_{t}^{i}=\left[s_{t-T+1}^{i}, s_{t-T+2}^{i}, \ldots, s_{t}^{i}\right]$,

式中, 变量 $s_{t}{ }^{i}$ 的表达式为

$s_{t}^{i}= \begin{cases}v_{t}^{x}, p_{t}{ }^{y}, & t \equiv 0\left(\bmod t_{\omega}\right), \\ v_{t}^{x}, & \text { 其他, }\end{cases}$

式中, $v_{t}^{x}$ 和 $p_{t}{ }^{y}$ 分别为 $t$ 时刻电力网络节点 $x$ 电压和天然 气网络节点 $y$ 压力, $t_{\omega}$ 为压力变量 $p_{t}{ }^{y}$ 的采样周期.

当网络中不同检测变量 $s^{i}$ 按照节点顺序依次排列
时, 检测序列 $\mathbf{s}_{t}^{i}$ 组成原始检测矩阵 $\mathbf{S}_{t}$ 并且其表达式为

$\mathbf{S}_{t}=\left[\Delta \mathbf{s}_{t}^{1}, \Delta \mathbf{s}_{t}^{2}, \ldots, \Delta \mathbf{s}_{t}^{i}, \ldots, \Delta \mathbf{s}_{t}^{n}\right]^{\mathrm{T}} \in \mathbb{R}^{n \times T}$,

式中, 检测向量 $\Delta \mathbf{s}_{t}^{i}=\left|\mathbf{s}_{t}^{i}-\mathbf{s}_{t-1}^{i}\right|$, 检测变量 $s^{i}$ 的数量为

$n= \begin{cases}n_{e}+n_{g}, & t \equiv 0\left(\bmod t_{\omega}\right), \\ n_{e}, & \text { 其他. }\end{cases}$

在此基础上, 考虑到电-气耦合网络的检测变量具 有不同的单位和变化度, 本节对原始检测矩阵 $\mathbf{S}$ 进行 归一化及数据转换处理, 使其能够在同一量级进行分 析, 转换过程及转换后的矩阵 $\mathbf{M}_{t}$ 定义为

$\mathbf{M}_{t}=\Gamma\left[\mathbf{S}_{t}\right]^{\mathrm{T}} \odot \Gamma\left[\ln \left(\mathbf{S}_{t}\right)+\boldsymbol{\alpha}\right]$,

式中, $\odot$ 为点乘运算, $\boldsymbol{\alpha}$ 为实常数 $\tilde{\alpha}$ 构成的列向量. 并且 归一化函数 $\Gamma[\cdot]$ 的表达式为

$\Gamma[\boldsymbol{\Psi}]=\frac{\boldsymbol{\Psi}-\min (\boldsymbol{\Psi})}{\max (\boldsymbol{\Psi})-\min (\boldsymbol{\Psi})}$,

式中, $\boldsymbol{\Psi}$ 为待归一化矩阵, $\min (\boldsymbol{\Psi})$ 为矩阵 $\boldsymbol{\Psi}$ 元素的最小 值, $\max (\boldsymbol{\Psi})$ 为矩阵 $\boldsymbol{\Psi}$ 元素的最大值.

进一步, 对矩阵 $\mathbf{M}_{t}$ 行向量 $\mathbf{m}_{t}$ 进行如下所示的标准 化转换, 得到标准化行向量 $\mathbf{m}_{t}$ :

$\widetilde{\mathbf{m}}_{t}=\frac{\mathbf{m}_{t}-\mu\left(\mathbf{m}_{t}\right)}{\sigma\left(\mathbf{m}_{t}\right)}$.

通过上式行向量 $\mathbf{m}_{t}$ 构建的矩阵, 即为本文提出的异构 数据模型 $\widetilde{\mathbf{M}}_{t}$.

\section{3 基于异构数据模型的状态感知算法}

根据第 2 节叙述可知, 异构数据模型 $\widetilde{\mathbf{M}}_{t}$ 是一个多 维矩阵. 为了适应不同网络节点特性和实际问题需求, 从随机矩阵理论出发, 本文在上一节构建的异构数据 模型基础上提出电-气耦合网络的状态感知方法, 为后 续网络的优化控制提供相应的信息依据.

\section{1 算法依据}

随机矩阵起源于 20 世纪 50 年代的核物理研究领 域, 并且围绕随机矩阵谱分布取得了一系列重大进展, 例如半圆律、单环定理以及马尔琴科-帕斯图尔定律 (Marčenko-Pastur Law, MP Law) ${ }^{[28,29]}$. 随着研究数据维 
度和数量不断增大, 随机矩阵可以在不进行数据降维 情况下完成对不适用于经典统计理论分析处理的多维 数据问题研究, 因此随机矩阵的谱理论逐渐应用于各 个多维数据研究领域.

在对矩阵谱分布极限研究的过程中, MP Law表明 当随机矩阵的维度比 $r$ 以相同速度趋于无穷大时, 协方 差矩阵特征值会以概率 1 收玫于固定分布 $F(x)$. MP Law的数学表达如下所示 ${ }^{[29]}$ :

$$
\begin{aligned}
\frac{\mathrm{d}}{\mathrm{d} x} & F(x) \\
& =\left\{\begin{array}{cc}
\frac{1}{2 \pi \sigma^{2} x r} \sqrt{(b-x)(x-a)}, & \text { 如果 } a \leq x \leq b, \\
0, & \text { 其他, }
\end{array}\right.
\end{aligned}
$$

式中, $\sigma^{2}$ 为协方差矩阵元素方差, $a=\sigma^{2}(1-\sqrt{r})^{2}$, $b=\sigma^{2}(1+\sqrt{r})^{2}$.

如图2(a)所示，当随机矩阵内元素为独立同分布 随机变量时, 随机矩阵的协方差矩阵谱分布近似于MP Law; 当矩阵元素不符合独立同分布时, 谱分布会偏离 理论MP Law范围, 并且会随着变化程度的不同而呈现 不同的谱分布情况，尤其是最大特征值变化最为明显， 具体谱分布情况如图2(b)所示.

\section{2 算法设计}

基于第3.1节描述可知, 异构数据模型 $\mathbb{\mathbf { M }}_{t}$ 为随机矩 阵, 并且进一步对其进行处理可以得到协方差矩阵 $\mathbf{C}_{t}$ :

$$
\mathbf{C}_{t}=\frac{1}{T} \widetilde{\mathbf{M}}_{t} \widetilde{\mathbf{M}}_{t}{ }^{\mathrm{T}}=\mathbf{U}_{t} \mathbf{E}_{t} \mathbf{U}_{t}{ }^{-1},
$$

式中, 矩阵 $\mathbf{E}_{t}$ 的对角线元素为协方差矩阵 $\mathbf{C}_{t}$ 的特征值,

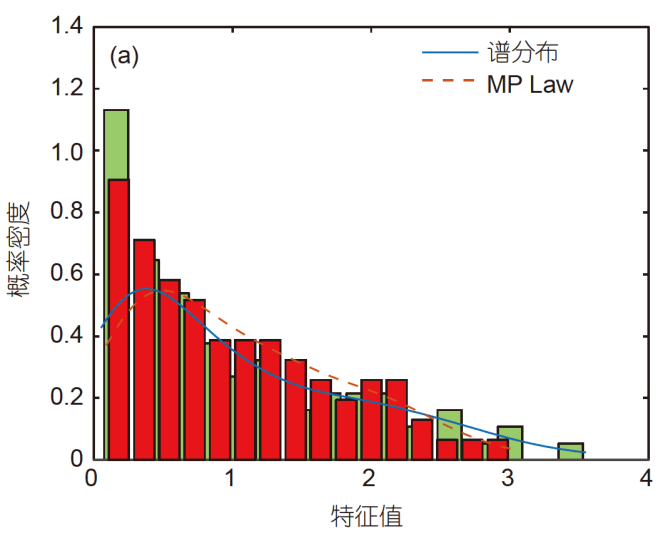

矩阵 $\mathbf{U}_{t}$ 为对应最大特征向量组成的矩阵.

通过 3.1节可知: 在得到协方差矩阵 $\mathbf{C}_{t}$ 的基础上, 通过MP Law可以反映检测变量的变化情况，协方差 矩阵 $\mathbf{C}_{t}$ 的谱分布可以充分反映检测序列的变化情况, 进而表明数据中存在非随机特性. 为了对谱分布情况 进行深入分析并且得到电-气耦合网络非随机变化情 况，本节提出衡量协方差矩阵 $\mathbf{C}_{t}$ 谱分布变化情况的公 式如下所示:

$$
\mathrm{P}_{t}=\operatorname{line}\left[J S\left(F_{t}^{\mathbf{C}} \| F^{\mathrm{H}}\right)\right] \text {, }
$$

式中, line [·]为线性变换函数, JS $(\cdot)$ 表示 JSS散度 (JensenShannon divergence)的变化情况, $F^{\mathrm{H}}$ 为随机矩阵理论 谱分布, $F_{t}^{\mathbf{C}}$ 为协方差矩阵 $\mathbf{C}_{t}$ 在 $t$ 时刻的谱分布.

此外在谱分布当中, 最大特征值 $\lambda_{t}{ }^{\max }$ 对于检测变 量 $s$ 的改变最为敏感. 同样地, 对最大特征值 $\lambda_{t}{ }^{\text {max 进行 }}$ 类似的处理, 得到最大特征值 $\lambda_{t}{ }^{\max }$ 的变化情况为

$\tilde{\lambda}_{t}^{\max }=\operatorname{line}\left[\lambda_{t}^{\max }\right]$,

式中, line[-]的含义同式(10), 具体变换函数形式根据 耦合网络情况确定.

协方差矩阵 $\mathbf{C}_{t}$ 的谱分布反映整体变化情况，而最

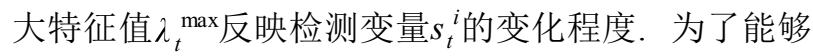
及时准确地感知电-气耦合网络状态, 需要从谱分布整 体和局部同时出发进行考虑，选取能够对不同程度的 变化出现不同程度改变的函数对两者的变化值进行统 一分析, 从而最终得到状态感知变化程度. 又因为双曲 正切函数会随着输入的微小变化而剧烈改变, 并且最

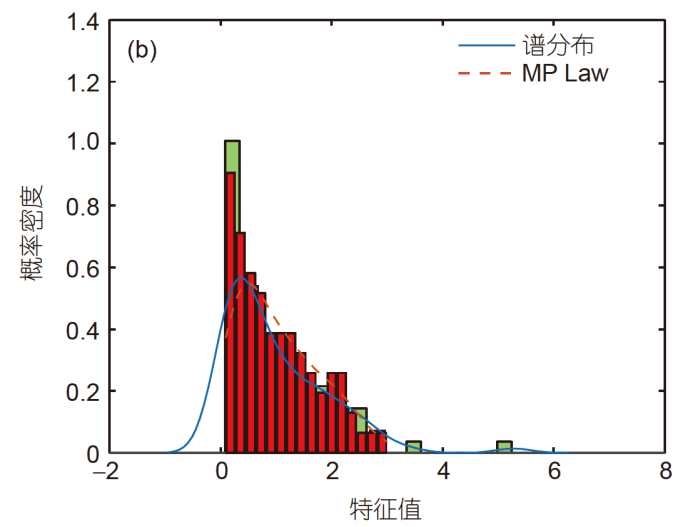

图 2 (网络版彩图)随机矩阵谱分布曲线. (a) 随机情况; (b) 非随机情况

Figure 2 (Color online) Spectral distribution curve of random matrix. (a) Random condition; (b) non-random condition. 
后逐渐平缓直至达到1. 也就是说, 双曲正切函数的数 学特性符合状态感知的需求, 因此本节选取双曲正切 函数表示状态感知变化程度, 同时定义 $t$ 时刻谱差异值 $d_{t}$ 为

$d_{t}=a_{1} \cdot \tanh \left(\eta \cdot P_{t}\right)+a_{1} \cdot \tanh \left(\eta \cdot \tilde{\lambda}_{t}^{\max }\right)$,

式中, $\tanh (\cdot)$ 为双曲正切函数, $a_{1}$ 和 $a_{2}$ 分别为谱分布及 最大特征值变化情况的权重系数, $\eta$ 为实常数.

进一步地, 根据谱差异值 $d_{t}$ 的变化情况, 表明 $t$ 时刻 耦合网络的状态变化程度 $D_{t}$, 具体划分情况如下所示:

$D_{t}= \begin{cases}\text { 状态无变化 }, & 0 \leq d_{t} \leq 0.4, \\ \text { 状态改变, } & 0.4<d_{t} .\end{cases}$

在得知电-气耦合网络内有状态改变时, 进一步通 过矩阵最大特征向量元素的值实现变化节点定位.

设 $t$ 时刻矩阵最大特征值 $\lambda_{t}{ }^{\max }$ 对应的特征向量 $\mathbf{U}_{t}^{\text {max }}$ 的元素为 $u_{t}{ }^{\text {max }}(i)(i=1,2, \ldots, n)$, 当 $u_{t}{ }^{\text {max }}(j)$ 满足下式时, 说 明此时元素 $u_{t}^{\text {max }}(j)$ 的值最大.

$u_{t}^{\max }(j)=\max \left\{u_{t}^{\max }(1), u_{t}^{\max }(2), \ldots, u_{t}^{\max }(n)\right\}$,

式中, $\max \{\cdot\}$ 为最大值函数.

接着根据式(14)得到变化节点的编号为

$l= \begin{cases}j, & 1 \leq j \leq n_{e}, \\ j-n_{e}, & n_{e}+1 \leq j \leq n_{e}+n_{g} .\end{cases}$

最后通过上式计算得到的编号可知变化的节点为 $s$.

当变化节点 $s$ 的改变影响整个电-气耦合网络安全 运行时, 电力网络的检测变量 (节点电压 $v^{x}$ )和天然气网 络的检测变量(节点压力 $p^{y}$ )会急剧改变, 严重影响网络 正常运行甚至会导致系统失稳情况. 因此在网络电/气 子系统调节裕度充足的情况下, 可以首先考虑发电机 的一次调压/调频、静止补偿器出力调整和天然气网 络气原调节补偿节点负荷所需, 解决系统网络状态改 变的问题. 当电-气耦合网络需要能量互济以保证系统 安全稳定运行时, 可以采用文献[10]所提出的基于可 变压缩比的电-气耦合网络稳定控制方法以保证整个 网络的安全稳定运行.

备注 由于状态变化程度 $D_{t}$ 决定着当前时刻电-气 耦合网络状态是否发生变化, 因此设定具体的划分范 围对于耦合网络状态感知的准确判断是十分重要的. 因此，本节通过理论与实际相结合的方式实现相应情
况的划分设定.

从理论角度分析, 本文提出的基于异构数据模型 的状态感知算法是通过协方差矩阵谱分布实现的. 当 电-气耦合网络的检测变量数据构建的异构数据模 型 $\mathbf{M}_{t}$ 实时满足随机矩阵随机特性时, 协方差矩阵 $\mathbf{C}_{t}$ 的 谱分布与随机矩阵理论谱分布 $F^{\mathrm{H}}$ 一致. 根据本文提出 的状态感知流程及式(10)和(11)可得, 谱分布变化 $P_{t}=0$, $\tilde{\lambda}_{t}^{\max }=0.075$. 接着依据式(12)计算可知, 谱差异值 $d_{t}=0.1106$. 所以, 在理想状态下状态变化程度 $D_{t}$ 在 0.1106 处进行划分.

从实际角度分析, 由于环境噪声、测量装置以及 数据传输等原因, 电-气耦合网络的检测变量数据通常 不会满足理论随机矩阵的要求, 也就是数据存在非随 机特性. 进而会导致协方差矩阵 $\mathbf{C}_{t}$ 的谱分布会与随机 矩阵理论谱分布不一致, 使得谱分布变化 $P_{t} \neq 0$ 并且最 大特征值变化 $\tilde{\lambda}_{t}{ }^{\max } \geq 0.075$, 所以谱差异值 $d_{t}$ 会大于理 论值 0.1106 .

为了进一步确立设定的状态变化程度, 对本文所研 究的电-气耦合网络进行了大量的仿真研究, 最终确定 0.4 为本文状态变化程度的划分值. 需要说明的是, 0.4 并 不是固定不变的, 是可以根据实际情况进行调整的.

综上所述，当 $t$ 时刻电-气耦合网络节点检测变量 $s^{i}$ (电力网络节点电压 $v^{x}$ 或者天然气网络节点压力 $p^{y}$ ) 的 数据发生改变时, 协方差矩阵 $\mathbf{C}_{t}$ 的谱分布会依照网络 节点变化情况发生不同程度的改变. 基于异构数据模 型的电-气耦合网络状态感知算法的步骤如图3所示.

\section{4 仿真算例分析}

在本文中, 将分成 3 个算例 4 种不同情况进行仿真 研究. 第 4.1 节及 4.2 节分别感知电力网络电负荷节点 和天然气网络气负荷节点的变化, 第4.3节对不同类型 的能量耦合设备进行状态感知研究, 分析本文提出的 方法对耦合设备改变的感知情况. 三个算例均采用如 图4所示的IEEE 33 节点以及天然气20节点网络 ${ }^{[30]}$ 构 成的电-气耦合网络及典型耦合设备进行仿真, 其具体 结构如图4所示, 具体能源设备耦合情况如表1所示.

根据电-气耦合网络结构可知: 检测变量 $s$ 的数量 $n=53$, 采样时间长度 $T=101$, 并且在下面仿真算例中, 压力变量 $p^{y}$ 的采样周期 $t_{\omega}=20 \mathrm{~s}$, 设置实常数 $\tilde{\alpha}=50$, 协 


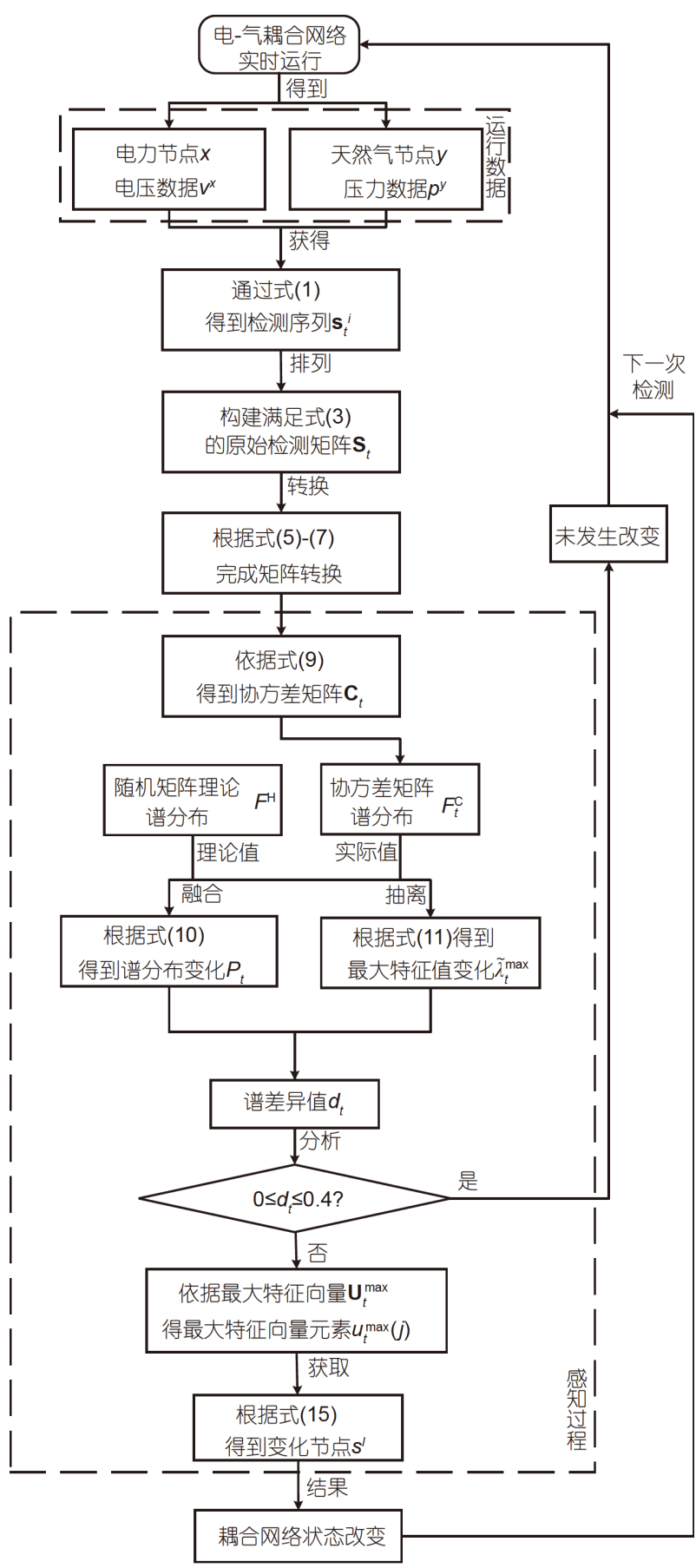

图 3 电-气耦合网络状态感知流程图

Figure 3 Flowchart of situation awareness of electricity-gas coupling network.

方差矩阵 $\mathbf{C}_{t}$ 谱分布和最大特征值 $\lambda_{t}{ }^{\max }$ 依据线性函数变 换得到的线性区间分别为 $[0,0.2]$ 和 $[0,20]$. 为了均衡考 量数据整体变化情况以及局部变化程度，设置权重系
数为 $a_{1}=a_{2}=0.5, \eta=3$.

\section{1 电网负荷变化算例分析}

为了研究不同电负荷变化对电-气耦合网络的影 响，本节设定同一节点的3种不同负荷变化进行研究. 为了便于进行不同变化程度的比较，不同负荷变化时 间均设置在同一采样时间点，具体节点及变化情况如 表2所示.

根据仿真算例设置参数可知，电力网络节点电压 $v^{x}$ 采样周期为 $1 \mathrm{~s}$, 天然气网络的节点压力 $p^{y}$ 的采样周 期 $t_{\omega}$ 为 $20 \mathrm{~s}$ ，所以在仿真中每隔 $20 \mathrm{~s}$ 用长尺度数据模型 进行一次状态感知检测. 在电力节点 25 不同负荷变化 下，不同时刻检测变量构建的协方差矩阵谱差异值变 化情况如图5所示.

为了表明状态感知算法的有效性，下面只选取电 负荷增加情况为例进行说明. 针对变化 1 来说，因为从 第 $1 \mathrm{~s}$ 就开始采集数据进行异构数据模型检测初始化, 并且默认第 $1 \mathrm{~s}$ 就用长尺度数据模型进行检测，所以当 负荷变化发生在第 $200 \mathrm{~s}$ 时，根据第 $201 \mathrm{~s}$ 异构数据模型 的协方差矩阵可知，谱差异值 $d_{t}$ 由 $200 \mathrm{~s}$ 的 0.1836 突然 上升至 $201 \mathrm{~s}$ 的 0.5449 , 超过了式(13)设置的检测阈值 0.4 ，并且第 $221 \mathrm{~s}$ 的谱差异值大于检测阈值 0.4 表明了 此时电-气耦合网络状态已经发生改变. 从图5的 3 种不 同变化纵向对比来看, 根据电负荷变化程度的不同, 谱 差异值会出现不同的变化并且均超过检测阈值0.4. 因 此，通过谱差异值可以准确地判断出耦合网络状态是 否改变. 并且通过图 5 及表 2 负荷变化情况可知，谱差 异值变化量会反映负荷变化程度，为后续的分析及操 作提供依据. 在算例仿真分析过程中，本算例设定的 电力网络节点、天然气网络节点变化的时间恰好是天 然气网络压力变量采样时间的倍数，同时假设算例仿 真中节点变化出现在采样时刻之后. 所以在节点参数 发生改变时，电力网络节点能够在下一采样时刻采集 到数据变化, 而天然气节点需要等到一个采样周期才 能采集到数据的变化, 进而采用长尺度数据模型完成 检测. 因此在仿真中会出现变化时间相同的情况.

在通过式(13)得知状态改变后，图6为通过谱分布 得到的表征耦合网络节点变化的最大特征向量元素, 其中图6(a) (c)分别为变化事件1 3的变化情况. 根据 图3所示流程图可知，当谱差异值达到设定阈值后，需 


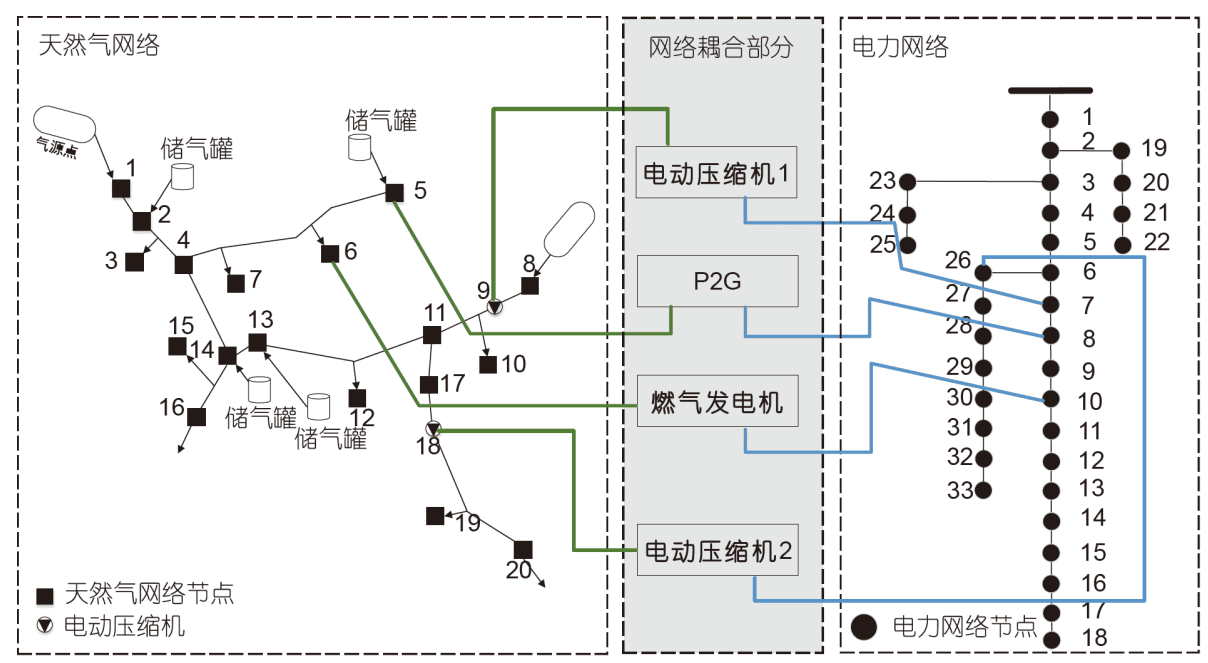

图 4 (网络版彩图)电-气耦合网络结构

Figure 4 (Color online) Electricity-gas coupling network structure.

表 1 电-气耦合节点情况

Table 1 Electricity-gas coupling node

\begin{tabular}{ccc}
\hline 耦合设备 & 电力网络节点 & 天然气网络节点 \\
\hline 燃气轮机 & 10 & 6 \\
$\mathrm{P} 2 \mathrm{G}$ & 8 & 5 \\
电动压缩机 1 & 7 & 9 \\
电动压缩机 2 & 26 & 18 \\
\hline
\end{tabular}

表 2 电负荷变化参数

Table 2 Parameters of electrical load change

\begin{tabular}{cccc}
\hline 变化 & 变化时间 $(\mathrm{s})$ & 变化电网节点 & 变化情况 \\
\hline \multirow{2}{*}{1} & 200 & & 负荷增加 $2.38 \%$ \\
& 500 & & 负荷恢复到正常值 \\
2 & 200 & 25 & 负荷增加 $23.81 \%$ \\
& 500 & & 负荷恢复到正常值 \\
3 & 200 & & 负荷增加 $119.05 \%$ \\
& 500 & 负荷恢复到正常值 \\
\hline
\end{tabular}

要通过最大特征向量 $\mathbf{U}_{221}^{\max }$ 确定变化节点. 并且通过计 算发现, $u_{221}^{\max }(25)$ 的值为 0.3257 并且满足式(14), 接着 通过式(15)得到变化节点编号为 25 , 也就是说变化节 点为电力网络节点 25 , 与表 2 中的变化节点相同. 通过 类似的方法及分析，可以得到其余两个变化节点均为 电力网络节点 25 . 综合上述分析过程可知, 本文提出
的方法能够准确地判断出状态改变情况并且实现变化 节点的定位.

\section{2 气网负荷变化算例分析}

相较于电力网络, 天然气网络的运行及时间尺度 等特性存在差异, 因此为了研究不同气负荷变化对电气耦合网络状态变化的影响, 本节设定同一节点的 3 种 不同负荷变化进行研究. 为了便于进行不同变化程度 的比较, 不同负荷变化时间均设置在同一采样时间点, 具体节点及变化情况如表3所示.

根据如图3所示的检测流程可得, 在天然气网络节 点20不同负荷变化下，不同时刻检测变量构建的协方 差矩阵谱差异值变化情况如图7所示.

为了表明状态感知算法对天然气节点负荷变化的 有效性，下面以第 $200 \mathrm{~s}$ 气负荷增加的情况为例进行说 明. 根据异构数据模型检测方法可知, 第 $1 \mathrm{~s}$ 能够同时 采集到电力网络节点电压和天然气网络节点压力数 据, 因此采用长尺度数据模型进行分析. 当第 $200 \mathrm{~s}$ 气 节点 20 负荷增加 $2.08 \%$, 由于算例仿真中节点变化出 现在采样时刻之后, 所以第 $200 \mathrm{~s}$ 的谱差异值 $d_{200}=$ $0.2324<0.4$. 第 $201 \mathrm{~s}$ 时电力网络节点电压变化较快, 所 以 $201 \mathrm{~s}$ 的异构数据模型谱差异值 $d_{t}$ 由 $200 \mathrm{~s}$ 的 0.2324 突 然上升至201 s的0.6603, 超过了式(13)设置的检测阈值 0.4 , 表明此时电-气耦合网络状态已经发生改变. 第 221 s 通过计算由电力网络节点电压和天然气节点压 


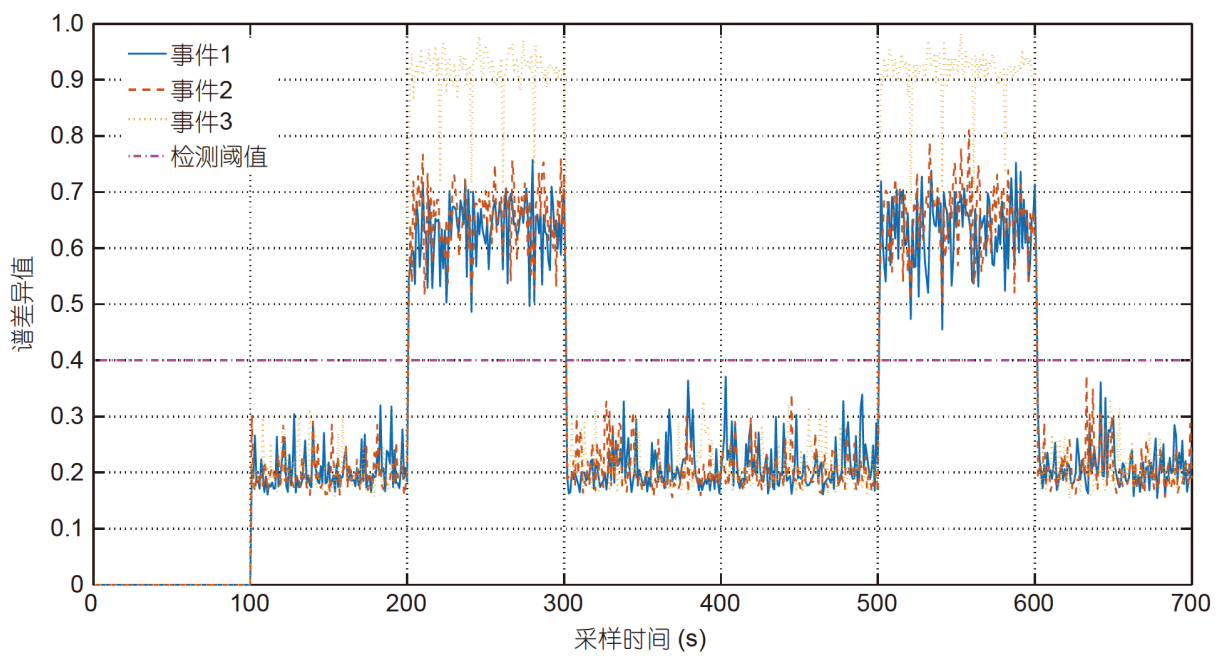

图 5 (网络版彩图)电负荷谱差异值曲线

Figure 5 (Color online) Spectral difference value curve of electrical load.
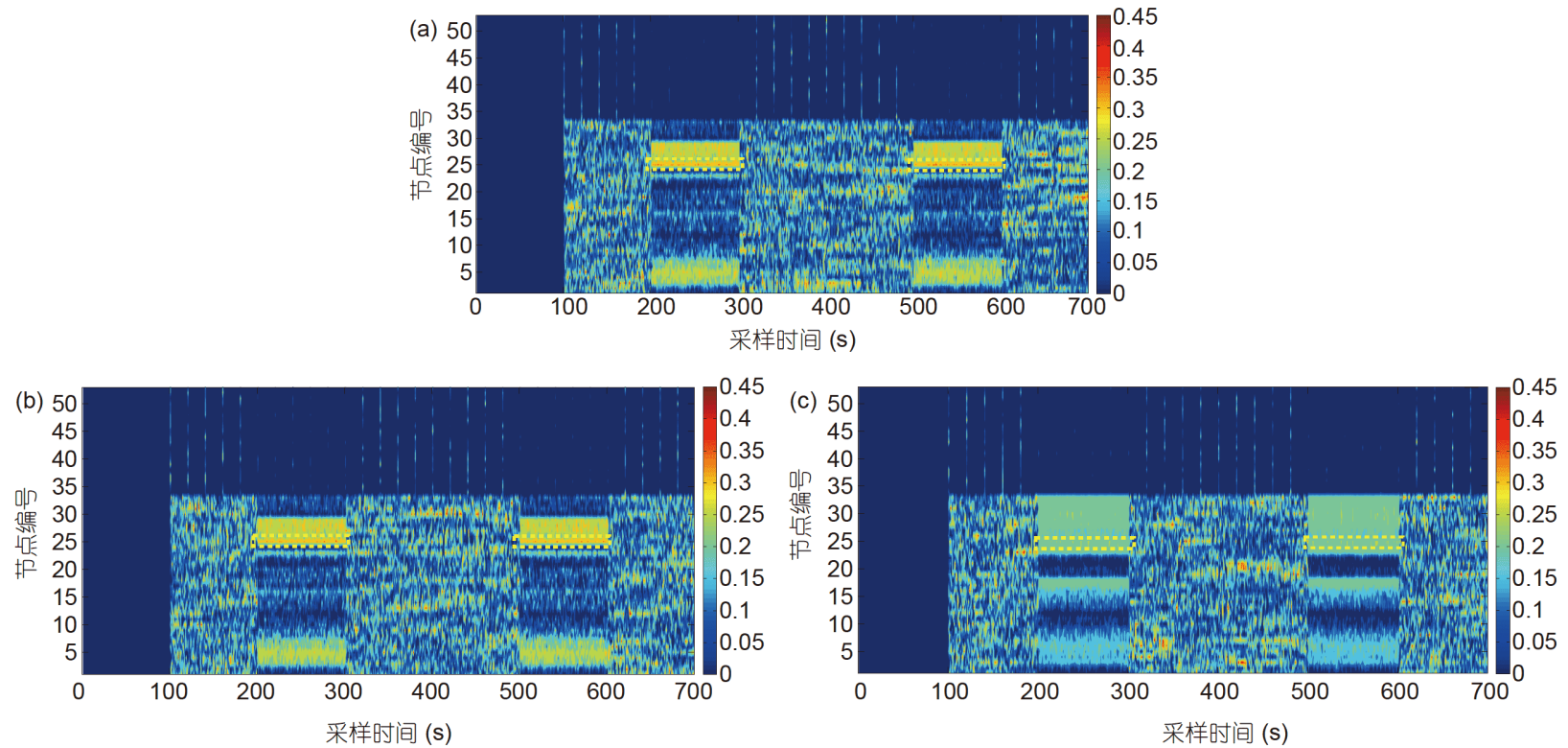

图 6 (网络版彩图)电负荷变化定位结果. (a) 电负荷变化1定位结果; (b) 电负荷变化2定位结果; (c) 电负荷变化3定位结果

Figure 6 (Color online) Location results of electrical load change. (a) Location result of electrical load change 1; (b) location result of electrical load change 2; (c) location result of electrical load change 3.

力数据组成的长尺度数据模型可知, 谱差异值 $d_{221}$ 为 0.6375 , 所以电-气耦合网络状态已经发生变化. 类似 地，通过上述过程也可以得到图7所示的其余变化情 况分析结果.

在已知电-气耦合网络状态发生改变的基础上, 图8为通过谱分布得到的表征耦合网络节点变化的最 大特征向量元素, 其中图 $8(a) \sim(c)$ 分别为电-气耦合网
络节点变化事件 1 3 的变化情况. 根据图3所示流程图 可知, 当谱差异值超过设定的检测阈值 0.4 后, 需要通 过最大特征向量 $\mathbf{U}_{221}^{\max }$ 确定变化节点. 通过计算发现, $u_{221}^{\max }(53)$ 的值为 0.2889 并且满足式(14), 接着通过 式(15)得到变化节点编号为53, 也就是说变化节点为 天然气网络节点 20 , 与表 3 中的变化节点相同. 通过相 似的判断方法, 可以得到其余两个变化节点均为天然 
表 3 气负荷变化参数

Table 3 Parameters of gas load change

\begin{tabular}{cccc}
\hline 变化 & 变化时间 $(\mathrm{s})$ & 变化气网节点 & 变化情况 \\
\hline \multirow{2}{*}{1} & 200 & & 负荷增加 $2.08 \%$ \\
& 500 & & 负荷恢复到正常值 \\
2 & 200 & \multirow{2}{*}{20} & 负荷增加 $4.69 \%$ \\
& 500 & & 负荷恢复到正常值 \\
3 & 200 & & 负荷增加7.30\% \\
& 500 & 负荷恢复到正常值 \\
\hline
\end{tabular}

气网络节点20. 综合上述分析过程可知, 本文提出的方 法能够准确地判断出天然气节点负荷改变引起的状态 改变情况并且实现变化气网节点的定位.

\section{3 电-气耦合网络相互作用算例分析}

电-气耦合网络中能量间的相互转化均依靠耦合 设备的传递, 如果耦合设备出现变化, 则会影响网络的 正常运行. 因此需要对耦合设备的微小变化进行实时 感知, 避免出现剧烈改变对整个网络运行及设备造成 不可逆的损害. 因此，本节主要考虑不同能量耦合设 备变化情况, 具体变化参数如表4及表 5 所示.

为了使描述更加清晰, 下面以变化 1 在 $200 \mathrm{~s}$ 变 化情况进行分析. 图9为 $\mathrm{P} 2 \mathrm{G}$ 出力改变的谱差异值曲 线. 在第1 199 s P2G保持稳定输出情况下, 电-气耦合 网络的谱差异值曲线没有超过检测阈值. 如表4所示,
当 $\mathrm{P} 2 \mathrm{G}$ 的出力在设定的第 $200 \mathrm{~s}$ 增加时, 由于电力网络 为快变系统, 因此第 $201 \mathrm{~s}$ 电力网络节点会及时发生改 变. 根据图3所示检测流程可得, 电-气耦合网络异构数 据模型的谱差异值 $d_{201}$ 为 0.7412 并且超过了预先设定 的检测阈值 0.4 , 说明此刻电力节点状态发生改变; 而 天然气网络的变化较慢, 所以会在天然气网络节点的 下一个采样周期也就是第221 s 获取整个天然气网络 节点的变化状态, 进而使用异构数据模型对整个电-气 耦合网络进行分析. 通过异构数据模型得到此时谱差 异值 $d_{221}$ 为 0.6522 , 因此天然气节点状态也发生改变. 同理，通过计算协方差矩阵谱分布及最大特征值得到 的图9谱差异值曲线可知, $\mathrm{P} 2 \mathrm{G}$ 在发生变化 2 和 3 情况下 的谱差异值均超过检测阈值 0.4 , 因此当前时刻网络状 态发生改变.

在此基础上，进一步根据最大特征向量 $\mathbf{U}_{221}^{\max }$ 判断 发生变化的节点. 通过图10(a)可知, 特征向量第8个元 素值最大, 所以最终得出变化节点为节点 8 . 通过对照 表4所设置的参数表明, 本文提出的方法能够有效地检 测P2G的变化情况. 通过类似的分析过程, 从图10(b) (c)及式(14)和(15)可得在200 s 发生变化2和变化3的节 点均是节点 8 . 综上所述, 在电-气耦合网络出现微小变 化的情况下, 基于异构数据的状态感知方法仍能够判 断正确.

除表4所述的 $\mathrm{P} 2 \mathrm{G}$ 耦合设备以外，气转电设备(燃 气轮机)在电-气耦合网络中承担着至关重要的作用,

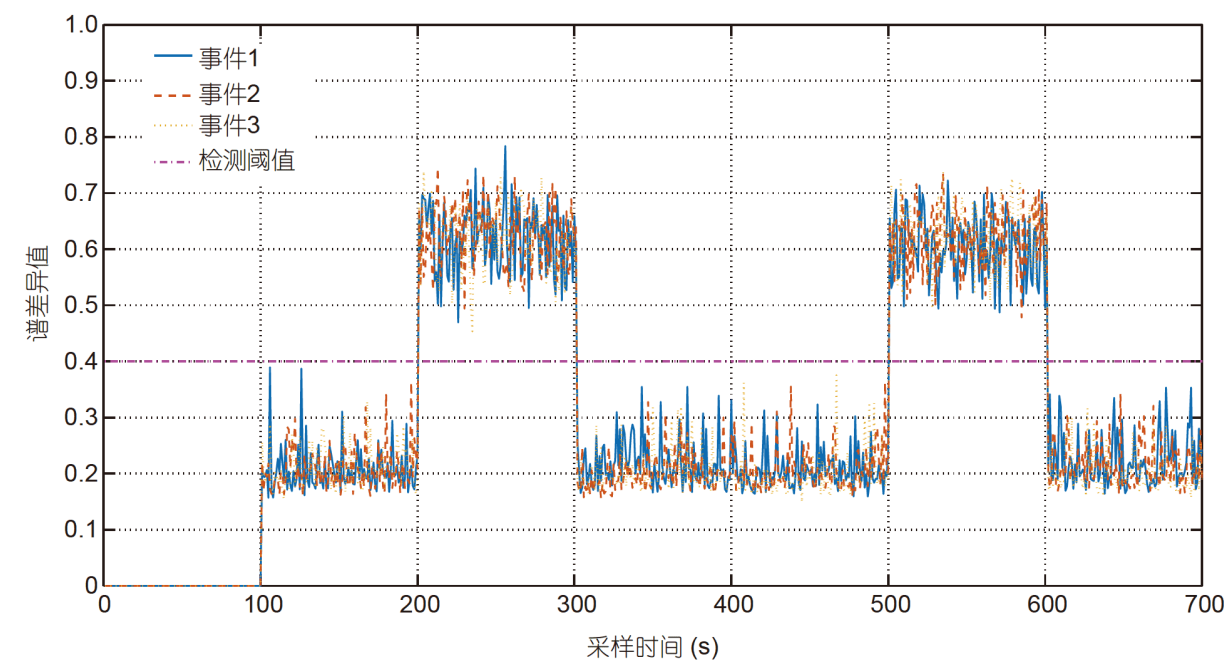

图 7 (网络版彩图)气负荷谱差异值曲线

Figure 7 (Color online) Spectral difference value curve of gas load. 

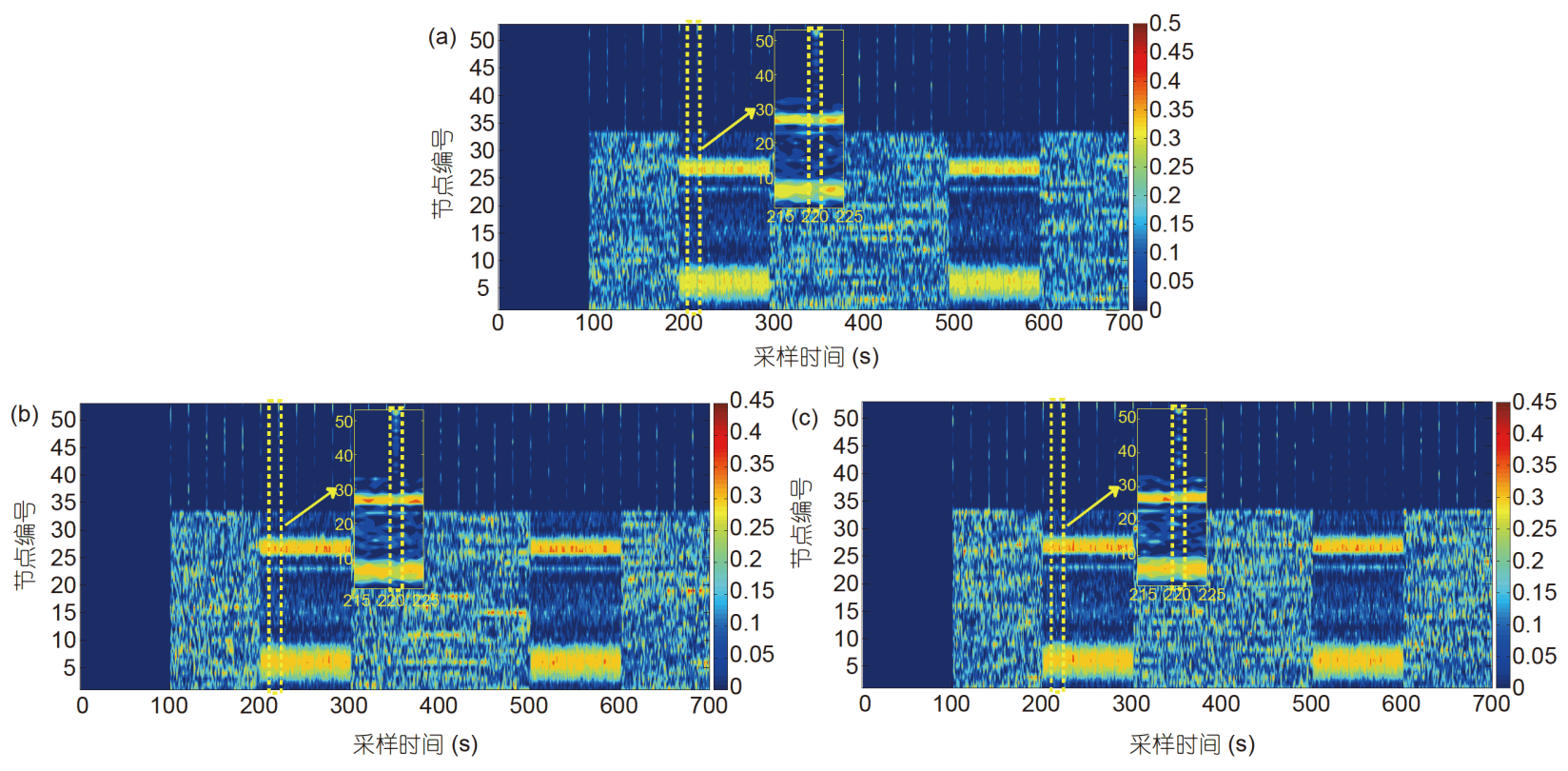

图 8 (网络版彩图)气负荷变化定位结果. (a) 气负荷变化1定位结果; (b) 气负荷变化 2 定位结果; (c) 气负荷变化3定位结果

Figure 8 (Color online) Location results of gas load change. (a) Location result of gas load change 1; (b) location result of gas load change 2; (c) location result of gas load change 3 .

表 4 P2G变化参数

Table 4 Parameters of $\mathrm{P} 2 \mathrm{G}$ change

\begin{tabular}{cccc}
\hline 变化 & 变化时间 $(\mathrm{s})$ & 变化电网节点 & 变化情况 \\
\hline \multirow{2}{*}{1} & 200 & & 负荷增加 $1.11 \%$ \\
& 500 & & 负荷恢复到正常值 \\
& 200 & \multirow{2}{*}{ 负荷增加 $5.56 \%$} \\
& 500 & & 负荷恢复到正常值 \\
& 200 & & 负荷增加 $11.11 \%$ \\
3 & 500 & & 负荷恢复到正常值 \\
\hline
\end{tabular}

保证电力的可靠供应. 因此本小节进一步研究了燃气 轮机的微小变化感知情况, 具体变化参数如表 5 所示.

如图11所示为燃气轮机出力变化时的谱差异值曲 线, 接下来为了分析燃气轮机参数变化引起的电-气耦 合网络电气节点状态变化情况，算例以变化 1 在第 $200 \mathrm{~s}$ 的出力改变情况为例进行描述. 在燃气轮机未增 加出力的时间段即采样时间为第1 199 s, 从图11所示 的变化 1 曲线中可以得到该时间段的谱差异值曲线在 未超过检测阈值的范围波动, 因此电-气耦合网络中的 节点状态并未发生明显变化. 当燃气轮机在设定的第 $200 \mathrm{~s}$ 出力增加 $0.50 \%$ 后, 在下一个采样时刻 $201 \mathrm{~s}$, 由于
表 5 燃气轮机变化参数

Table 5 Parameters of gas turbine change

\begin{tabular}{cccc}
\hline 变化 & 变化时间 $(\mathrm{s})$ & 变化气网节点 & 变化情况 \\
\hline 1 & 200 & & 出力增加 $0.50 \%$ \\
& 500 & & 出力恢复到正常值 \\
& 200 & 6 & 出力增加 $1.24 \%$ \\
2 & 500 & & 出力恢复到正常值 \\
& 200 & & 出力增加 $2.48 \%$ \\
3 & 500 & 出力恢复到正常值 \\
\hline
\end{tabular}

电力网络节点的电压最先发生改变, 因此根据异构数 据模型得到谱差异值 $d_{201}=0.716>0.4$, 所以判断网络状 态发生改变. 然后在第 $221 \mathrm{~s}$ 时, 通过电力节点电压和 天然气节点压力组成的长尺度数据模型分析, 谱差异 值 $d_{221}=0.4255$ 依然大于检测阈值 0.4. 因此耦合网络中 电气节点均已发生变化. 通过如图3所示的状态感知 流程图, 可以得到其余不同时刻燃气轮机的谱差异值 变化曲线. 根据图11可知: 当不同程度微小变化发生 在天然气网络节点 6 时, 本文提出的异构数据模型均 能够及时感知数据存在的非随机特性并且以谱差异值 的形式呈现出来. 


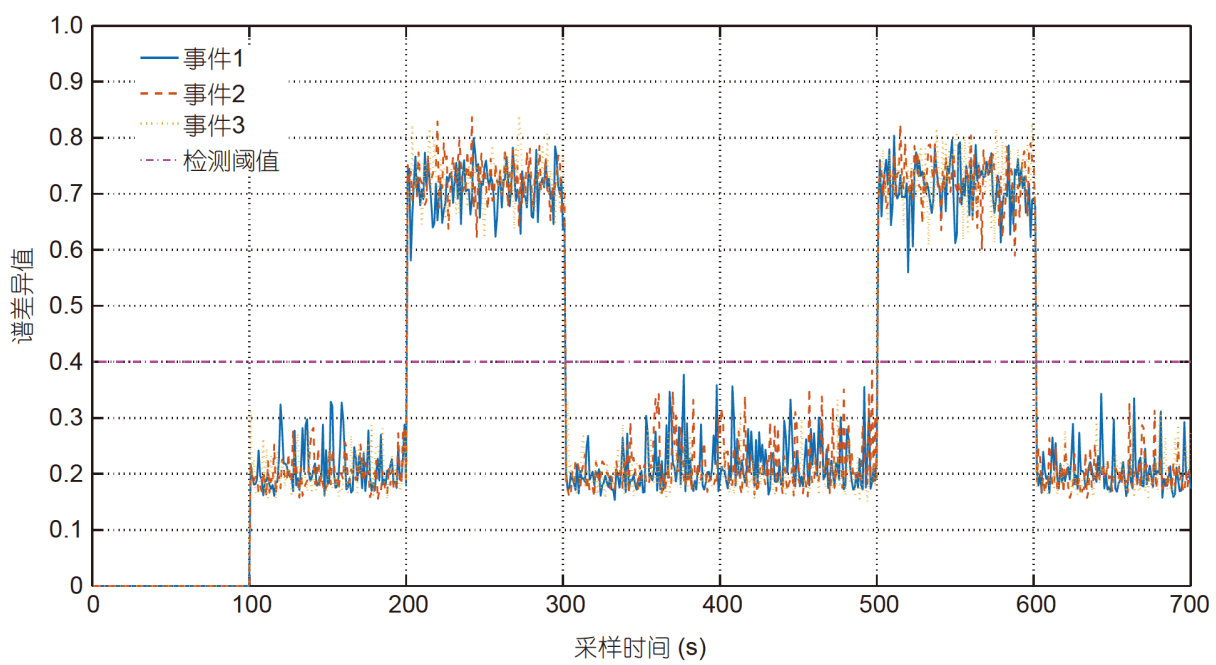

图 9 (网络版彩图)P2G出力谱差异值曲线

Figure 9 (Color online) Spectral difference value curve of P2G output.
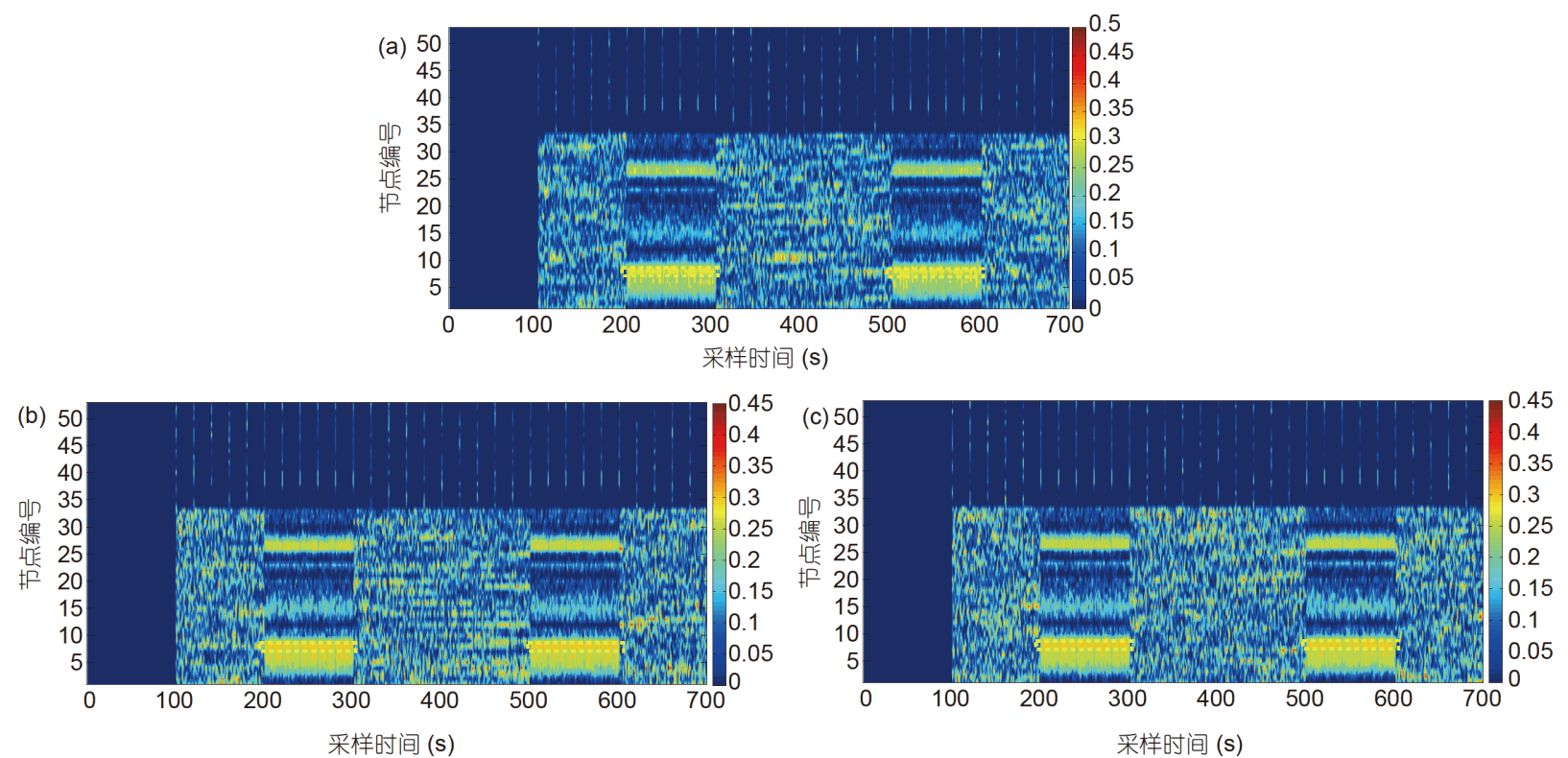

图 10 (网络版彩图)P2G出力变化定位结果. (a) P2G出力变化1定位结果; (b) P2G出力变化2定位结果; (c) P2G出力变化3定位 结果

Figure 10 (Color online) Location results of P2G output. (a) Location result of P2G output change 1; (b) location result of P2G output change 2; (c) location result of $\mathrm{P} 2 \mathrm{G}$ output change 3 .

图12均为燃气轮机变化定位结果. 进一步地, 为了 能够叙述清楚算法变化节点的定位过程，不失一般性， 下面仍然以 $200 \mathrm{~s}$ 变化情况为例进行说明. 当 $221 \mathrm{~s}$ 发现 电-气耦合网络出现变化情况后，通过异构数据模型的 协方差矩阵谱分布得到最大特征向量 $\mathbf{U}_{221}^{\max }$ 进行变化节 点的确定. 根据式(14)及图10(a)的局部放大图可知, 特征
向量元素 $u_{221}^{\max }(39)$ 的数值最大, 并且依照式(15)最终得到 变化节点为气网节点 6 , 也就是燃气轮机. 通过类似的定 位分析过程，从图12(b)和(c)的局部放大图及式(14)和 (15)可得, 在 $200 \mathrm{~s}$ 发生的变化 2 和 3 均是由气网节点 6 引起 的. 综上所述, 在燃气轮机出现微小变换的情况下, 本文 提出的方法仍能够准确地判断出对应的变化情况. 


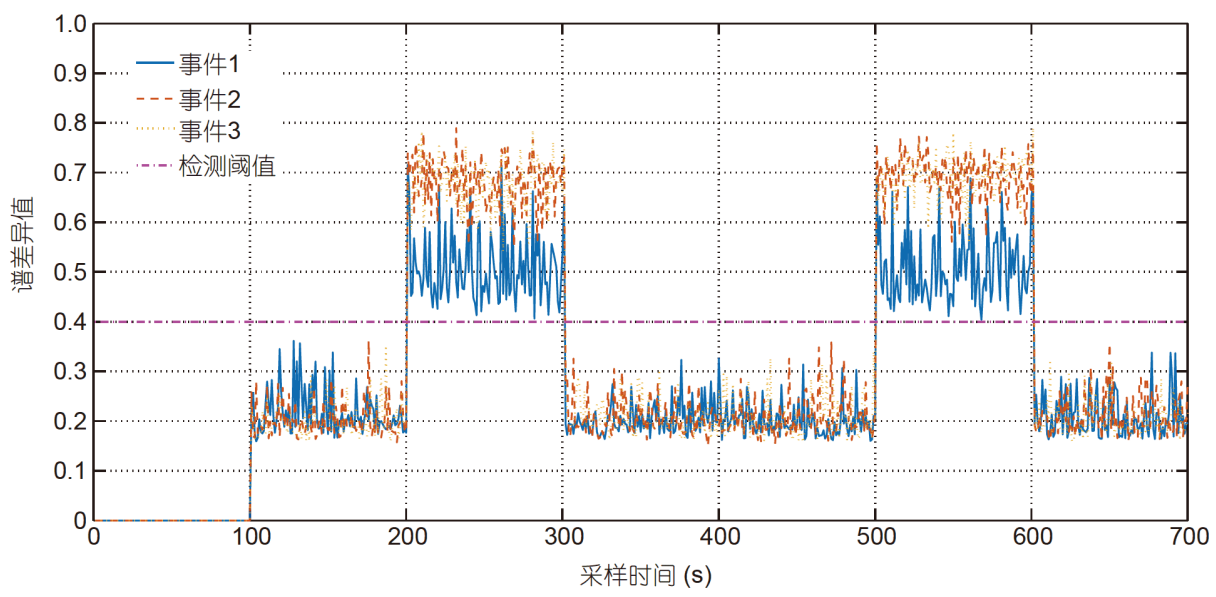

图 11 (网络版彩图)燃气轮机出力谱差异值曲线

Figure 11 (Color online) Spectral difference value curve of gas turbine output.
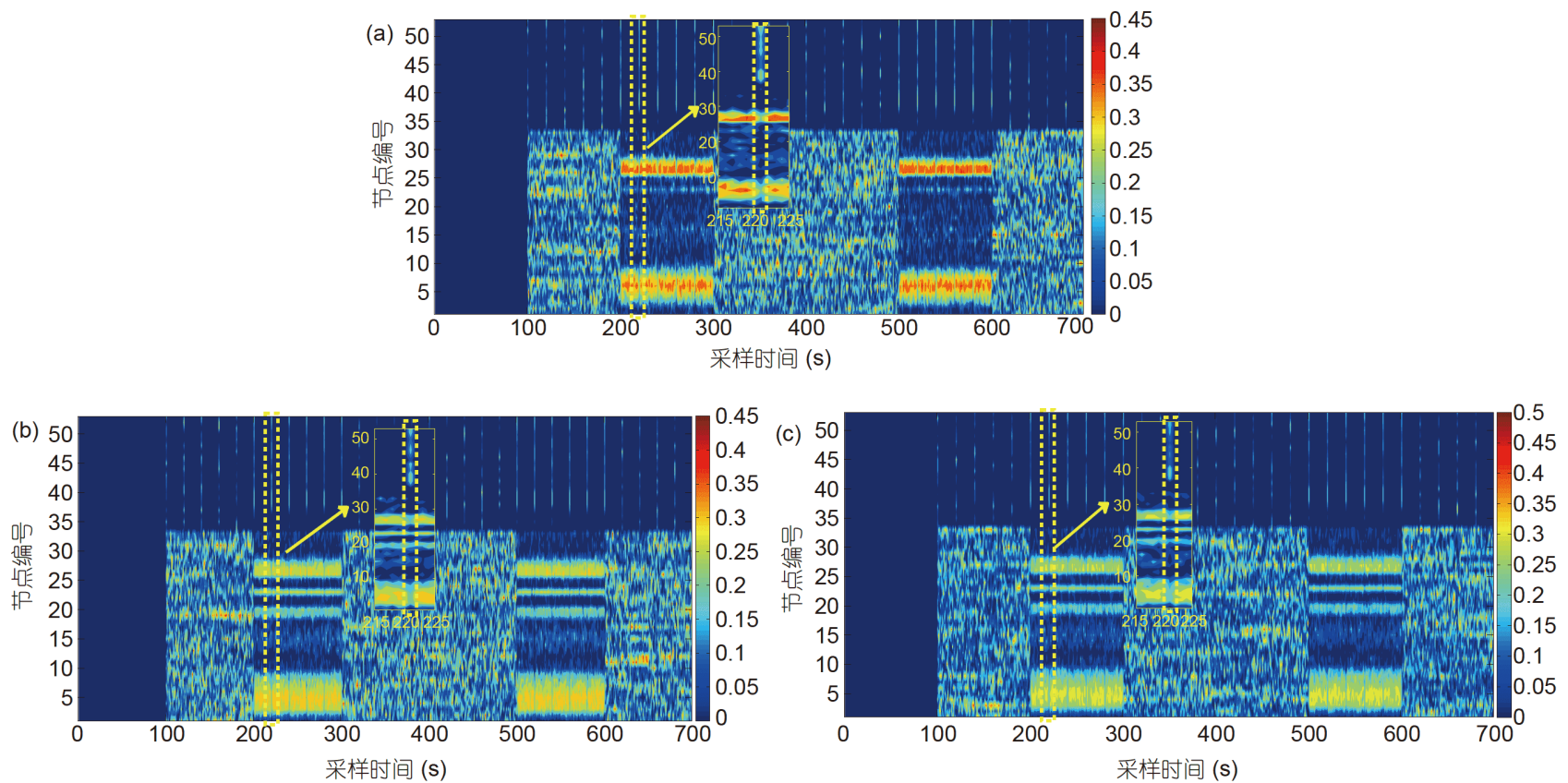

图 12 (网络版彩图)燃气轮机出力变化定位结果. (a) 燃气轮机出力变化1定位结果; (b) 燃气轮机出力变化 2 定位结果; (c) 燃 气轮机出力变化3定位结果

Figure 12 (Color online) Location results of gas turbine output. (a) Location result of gas turbine output change 1; (b) location result of gas turbine output change 2; (c) location result of gas turbine output change 3.

\section{5 结论}

针对电-气耦合网络面临的状态感知问题, 本文提 出一种基于异构数据模型的分析方法. 该方法首先建 立具有多时间尺度特性的异构数据模型，使其在减少
计算量的基础上能够有效地反映不同网络自身及相互 间的影响关系. 然后通过检测模型构建的协方差矩阵, 采用矩阵谱分布完成不同节点变化的状态感知，同时 引入与之对应的矩阵最大特征向量元素进行变化节点 的判断. 最后通过不同情况下的仿真结果表明该方法 
的可行性与有效性.

在电-气耦合网络运行过程中, 不同的变化节点会引 起其余节点数据的改变. 在检测变量发生变化的前提下, 本文所提方法能够检测出多个节点负荷发生变化. 如果 耦合网络内多个相近节点负荷同时变化会使节点间的 影响叠加, 使得节点检测变量数据变化更加复杂, 有时候 甚至会使作用在同一节点的变化相互抵消. 在此情形下, 虽然所提方法不能够准确感知变化节点位置, 但是根据
检测变量的变化情况会得到相应变化节点所在区域. 如 果网络内多个节点间的负荷变化影响不会叠加在相同 节点或者是多节点负荷变化影响不会在同一节点有明 显叠加效果, 那么本文提出的方法依然有效. 因此, 在未 来的研究中, 将会对电-气耦合网络数据进行特征分析并 且进一步研究节点数据变化机理, 将其与实际负荷变化 位置定位相结合，考虑不同节点负荷变化叠加作用的影 响, 进一步探索和改进所提出的状态感知方法.

\section{参考文献}

1 Sun Q Y, Teng F, Zhang H G. Energy internet and its key control issues (in Chinese). Acta Autom Sin, 2017, 43: 176-194 [孙秋野, 滕菲, 张化光. 能源互联网及其关键控制问题. 自动化学报, 2017, 43: 176-194]

2 Zhang H, Li Y, Gao D W, et al. Distributed optimal energy management for energy internet. IEEE Trans Ind Inf, 2017, 13: 3081-3097

3 Zhou X X, Zeng R, Gao F, et al. Development status and prospects of the energy internet (in Chinese). Sci Sin-Inform, 2017, 47: 149-170 [周孝 信, 曾嵘, 高峰, 等. 能源互联网的发展现状与展望. 中国科学: 信息科学, 2017, 47: 149-170]

4 Yang Z J, Gao C W, Zhao M. Review of coupled system between power and natural gas network (in Chinese). Autom Electric Power Syst, 2018, 42: 21-31, 56 [杨自娟, 高赐威, 赵明. 电力-天然气网络耦合系统研究综述. 电力系统自动化, 2018, 42: 21-31, 56]

5 Sun Q Y, Hu J W, Zhang H G. Modeling and application of we-energy in energy internet (in Chinese). Sci Sin-Inform, 2018, 48: 1409-1429 [孙 秋野, 胡旌伟, 张化光. 能源互联网中自能源的建模与应用. 中国科学: 信息科学, 2018, 48: 1409-1429]

6 Sun H B, Pan Z G, Guo Q L. Energy management for multi-energy flow: Challenges and prospects (in Chinese). Autom Electric Power Syst, 2016: 40: 1-8 [孙宏斌, 潘昭光, 郭庆来. 多能流能量管理研究: 挑战与展望. 电力系统自动化, 2016, 40: 1-8]

7 Wang C S, Wang D, Li L C, et al. Key technology analysis of demand-side smart energy system (in Chinese). Strat Study CAE, 2018, 20: 132140 [王成山, 王丹, 李立浧, 等. 需求侧智慧能源系统关键技术分析. 中国工程科学, 2018, 20: 132-140]

8 Xu X, Jia H, Wang D, et al. Hierarchical energy management system for multi-source multi-product microgrids. Renew Energy, 2015, 78: 621630

$9 \mathrm{Xu} \mathrm{X}$, Jia H, Chiang H D, et al. Dynamic modeling and interaction of hybrid natural gas and electricity supply system in microgrid. IEEE Trans Power Syst, 2015, 30: 1212-1221

10 Sun Q Y, Yu X T, Wang R, et al. Research on stability control strategy of electricity-gas coupling network based on dynamic model of natural gas network (in Chinese). Sci Sin-Tech, 2019, 49: 479-490 [孙秋野, 于晓婷, 王睿, 等. 基于天然气动态模型的电-气耦合网络稳定控制策略研究. 中国科学: 技术科学, 2019, 49: 479-490]

11 Wang W L, Wang D, Jia H J, et al. Review of steady-state analysis of typical regional integrated energy system under the background of energy internet (in Chinese). Proc CSEE, 2016, 36: 3292-3305 [王伟亮, 王丹, 贾宏杰, 等. 能源互联网背景下的典型区域综合能源系统稳态分析研 究综述. 中国电机工程学报, 2016, 36: 3292-3305]

12 Wang W L, Wang D, Jia H J, et al. Steady state analysis of electricity-gas regional integrated energy system with consideration of NGS network status (in Chinese). Proc CSEE, 2017, 37: 1293-1304 [王伟亮, 王丹, 贾宏杰, 等. 考虑天然气网络状态的电力-天然气区域综合能源系统稳态 分析. 中国电机工程学报, 2017, 37: 1293-1304]

13 Dong J N, Sun H B, Guo Q L, et al. State estimation of combined electric-gas networks for energy internet (in Chinese). Power Syst Tech, 2018, 42: 400-408 [董今妮, 孙宏斌, 郭庆来, 等. 面向能源互联网的电-气耦合网络状态估计技术. 电网技术, 2018, 42: 400-408]

14 Martinez-Mares A, Fuerte-Esquivel C R. A unified gas and power flow analysis in natural gas and electricity coupled networks. IEEE Trans Power Syst, 2012, 27: 2156-2166

15 Zhang Y B. Study on the methods for analyzing combined gas and electricity networks (in Chinese). Dissertation for Doctoral Degree. Beijing: China Electric Power Research Institute, 2005 [张义斌. 天然气-电力混合系统分析方法研究. 博士学位论文. 北京: 中国电力科学研究院, 2005] 
16 Shabanpour-Haghighi A, Seifi A R. An integrated steady-state operation assessment of electrical, natural gas, and district heating networks. IEEE Trans Power Syst, 2016, 31: 3636-3647

17 Zhao X, Yang L, Qu X B, et al. An improved energy flow calculation method for integrated electricity and natural gas system (in Chinese). Trans China Electrotech Soc, 2018, 33: 467-477 [赵霞, 杨仑, 篗小斌, 等. 电-气综合能源系统能流计算的改进方法. 电工技术学报, 2018, 33: 467477]

18 Zheng S L, Liu J, Chen Y B, et al. Bilinear robust state estimation bases on weighted least absolute value for integrated electricity-gas system (in Chinese). Power Syst Tech, 2019, 1-14 [郑顺林, 刘进, 陈艳波, 等. 基于加权最小绝对值的电-气综合能源系统双线性抗差状态估计. 电网技 术, 2019, 1-14]

19 Wang D, Liu L, Jia H, et al. Review of key problems related to integrated energy distribution systems. CSEE J Power Energy Syst, 2018, 4: 130145

20 Li T, Eremia M, Shahidehpour M. Interdependency of natural gas network and power system security. IEEE Trans Power Syst, 2008, 23: 18171824

21 Hibbard P J, Schatzki T. The interdependence of electricity and natural gas: Current factors and future prospects. Electr J, 2012, 25: 6-17

22 Chertkov M, Backhaus S, Lebedev V. Cascading of fluctuations in interdependent energy infrastructures: Gas-grid coupling. Appl Energy, 2015, 160: $541-551$

23 Devlin J, Li K, Higgins P, et al. A multi vector energy analysis for interconnected power and gas systems. Appl Energy, 2017, 192: 315-328

24 Luo B F, Mu Y F, Zhao B, et al. Static sensitivity analysis of integrated electricity and gas system based on unified power flow model (in Chinese). Autom Electric Power Syst, 2018, 42: 29-35 [骆柏锋, 穆云飞, 赵波, 等. 基于统一潮流模型的电-气耦合综合能源系统静态灵敏度 分析. 电力系统自动化, 2018, 42: 29-35]

25 Xin S, Guo Q, Sun H, et al. Cyber-physical modeling and cyber-contingency assessment of hierarchical control systems. IEEE Trans Smart Grid, 2015, 6: 2375-2385

26 Guo Q L, Xin S J, Sun H B, et al. Power system cyber-physical modelling and security assessment: Motivation and ideas (in Chinese). Proc CSEE, 2016, 36: 1481-1489 [郭庆来, 辛蜀骏, 孙宏斌, 等. 电力系统信息物理融合建模与综合安全评估: 驱动力与研究构想. 中国电机工程 学报, 2016, 36: 1481-1489]

27 Zhang H G, Wang Y C, Song Z. Absolute stabilization of singular systems with ferromagnetic hysteresis nonlinearity. Sci China Inf Sci, 2013, 56: 078201

28 Bai Z, Silverstein J. Spectral Analysis of Large Dimensional Random Matrices. 2nd ed. New York: Springer, 2010

29 Bai Z D, Zheng S R, Jiang D D. Large Dimensional Statistical Analysis (in Chinese). Beijing: Higher Education Press, 2012 [白志东, 郑术蓉, 姜 丹丹. 大维统计分析. 北京: 高等教育出版社, 2012]

30 De Wolf D, Smeers Y. The gas transmission problem solved by an extension of the simplex algorithm. Manag Sci, 2000, 46: 1454-1465 


\title{
Research on situation awareness of electricity-gas coupling network based on a heterogeneous data model
}

\author{
HU XuGuang ${ }^{1}$, ZHANG HuaGuang ${ }^{1,2}$, SUN QiuYe ${ }^{1,2}$, WANG Rui ${ }^{1}$ \& MA DaZhong ${ }^{1}$ \\ ${ }^{1}$ College of Information Science and Engineering, Northeastern University, Shenyang 110819, China; \\ ${ }^{2}$ State Key Laboratory of Synthetical Automation for Process Industries, Northeastern University, Shenyang 110819, China
}

Open interaction between the power system and the natural gas network, realizing the multi-energy flow, not only brings scientific management and optimal operation of energy but also increases the complexity of situation awareness due to energy conversion between independent energy networks. Therefore, this paper proposes a heterogeneous data model-based method embedding the characteristics of a coupling network for situation awareness of the multi-dimensional electricity-gas coupling network to achieve rapid analysis. First, considering the interaction between subnetworks and the change in the corresponding detection variables, the heterogeneous data model is developed to depict the time response characteristics of different subnetworks. Then, by the normalization and functional transform by the proposed model, a covariance matrix is obtained to improve the sensitivity to the nodedata change. Furthermore, the situation awareness of a coupling network is transformed into the degree analysis regarding the spectral difference value of a random matrix. Moreover, the changed node is obtained from the maximum eigenvector value of spectral distribution using the network situation change, completing the process of situation awareness. Finally, the feasibility and effectiveness of the proposed method are verified by different situation changes in the electricity-gas coupling network.

electricity-gas coupling network, multi-time scale, heterogeneous data model, data driven, spectral analysis 